NASA-CR-196011

A Search for Radiative Neutrino Decay from Supernovae

NAG 5-2062

Final Technical Report

$18 P$

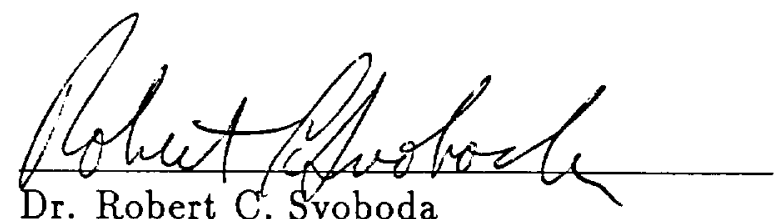

Dr. Robert C. Svoboda

Dept. of Physics and Astronomy

Louisiana State University

Baton Rouge, LA 70803-4001

(NASA-CR-196011) A SEARCH FOR

RADIATIVE NEUTRINO DECAY FROM

$N 94-35515$

SUPERNOVAE Final Technical Report

(Louisiana State Univ.) $18 \mathrm{p}$

Unclas

$63 / 930012611$ 


\begin{abstract}
Two supernovae have been identified in the COMPTEL data base as being the best sources to investigate for evidence of gamma-ray emission caused by radiative neutrino decay. These are SN1987a and SN1993J. A detailed simulation has shown us that we can expect a gain in sensitivity 1-3 orders of magnitude (depending on neutrino mass) over previous results. Instrument response is now being modeled using a SPARC10 computer aquired for this study. A library of simulated gamma-ray lines is being produced for COMPTEL as a by-product of this effort. Final results will be the Ph.D. thesis of R.Miller.
\end{abstract}




\section{Computer Simulation of Supernovae Neutrino Production}

A full 3-D computer simulation model of neutrino transport from an expanding spherically symmetric shell has been completed which includes the effects of a twobody neutrino decay with a single gamma in the final state. The original model used in the proposal has been corrected for phase-space effects in the assumed Fermi gas model of neutrino emission and also has been speeded up considerably by the use of approximations where appropriate. The model is now running at the COMPTEL site at $\mathrm{UNH}$ and has been used to make preliminary predictions of the gamma-ray flux at earth as a function of neutrino mass and lifetime.

In the analysis we have concentrated on two important type-II supernovae, SN1987A and SN1993J. The closeness of SN1987A means that heavy neutrinos have not yet reached earth but are still decaying on route at detectable fluxes. SN1993J, on the other hand, was much farther away $(3.2 \mathrm{Mpc})$ but COMPTEL observed it at a much earlier time so that intermediate masses would still be detectable. Figure 1 shows the expected sensitivity of COMPTEL from observations made during phase I and II. Also shown are the published limits from Solar Max observations of SN1987a in 1987. From this figure it is clear that a gain of 1-3 orders of magnitude in neutrino lifetime sensitivity will be achieved by analysis of COMPTEL data from these two supernovae. More detailed and advanced analysis techniques should improve the lifetime limits by 1-3 additional orders of magnitude. These results were presented at the Compton Symposium in St. Louis, the Cosmic Ray School at Erice, and at a GSFC colloquium.

\section{Computer Simulation of COMPTEL Response}

In order to perform a sensitive search for neutrino decay it is necessary to understand the COMPTEL instrument response to such a signature. This search covers a large portion of neutrino mass/lifetime parameter space and therefore requires many different gamma spectra to be compared with actual observational data. Because the COMPTEL instrument response to a given spectrum requires large amounts of CPU time, a method has been developed to synthesize the continuum gamma ray flux expected from neutrino decay using a library of mono-energetic instrument response lines. With the appropriate weighting factors the lines are combined into an instrument response which simulates that expected from an actual decay gamma ray spectrum. This instrument response library is currently under development at the University of New Hampshire. Once this library is complete, detailed analysis on the search for a radiative decay signature can begin.

\section{People and Equipment}

Richard Miller, who earned his M.S. at LSU has now moved to the University 


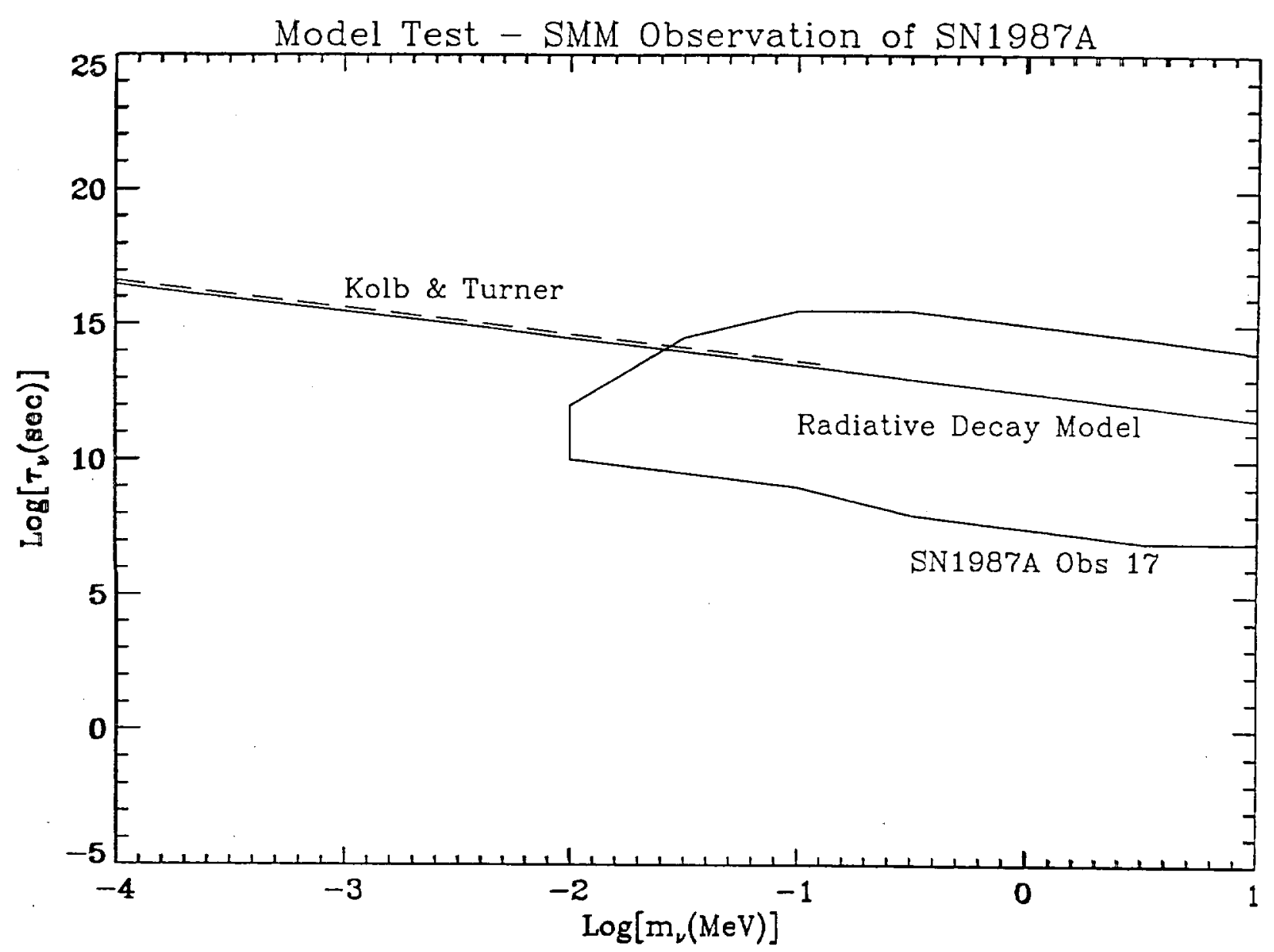

Figure 1:

of New Hampshire, where he is plans to finish his Ph.D. on analysis of SN1987A and SN1993J for neutrino decay. He is now working full-time on this with a NASAsponsored fellowship. Due to the computer-intensive nature of the COMPTEL instrument response, it was necessary to obtain a much faster computer than was available at $\mathrm{UNH}$ in order to complete the simulation work on the time scale of a Ph.D. thesis. Therefore, a SUN Sparc-10 was obtained through the Guest Investigator grant and dedicated to this analysis. The machine is now in place and being used by Miller at UNH.

\section{Talks Given}

Talks on radiative neutrino decay from supernovae were given at:

1. The Compton Symposium, St. Louis, 1992. (by R.Miller)

2. The Cosmic Ray School at Erice, June 1992. (by R.Miller)

3. GSFC Fellowship Seminar, September 1993. (by R.Miller) 
4. LSU Space Science Seminar, March 1994. (by R. Svoboda)

\section{Papers Published}

Published papers of the radiative neutrino decay analysis are:

1. "A Search for Radiative Neutrino Decay from Supernovae", The Compton Symposium, St. Louis, 1992, ed. M.Friedlander, N.Gehrels, and D.Macomb, AIP Conference Proceedings 280 pp 153-158, (1992).

2. "A Search for Radiative Neutrino Decay from Supernovae", Proceedings of the NATO Advanced Study Institute on Particle Astrophysics and Cosmology, ed. M.M.Shapiro, R.Silberberg, and J.P.Wefel, pp 111-118, (1993). 


\title{
A SEARCH FOR RADIATIVE NEUTRINO DECAY FROM SUPERNOVAE
}

\author{
R.S. Miller and R.C. Svoboda \\ Department of Physics and Astronomy \\ Louisiana State University \\ Baton Rouge, LA 70803-4001
}

\begin{abstract}
If a massive neutrino species exists then it is possible that it has a radiative decay mode. Cosmological arguments require that a neutrino of mass $>100 \mathrm{eV}$ be unstable. Motivated by these considerations, a 3-D model of radiative neutrino decay has been developed and used to simulate the decay evolution of supernova neutrinos. A sensitive search for the decay gamma rays using extragalactic supernovae and SN1987a as neutrino sources is proceeding using the COMPTEL instrument aboard the Compton Gamma Ray Observatory.
\end{abstract}

\section{The Search for Massive Neutrinos}

The search for the existence of a massive neutrino species is an area of intense theoretical and experimental research. Until recently, laboratory studies indicated that the observed properties of the neutrino were consistent with zero rest mass. The notable exceptions have been the experiments studying solar neutrinos and nuclear beta decay [1].

Very massive neutrinos ( $>100 \mathrm{eV}$ ) are expected to be unstable. Astrophysical limits based on the requirement that the mass density of the universe not exceed $\Omega=1$ require $\Sigma m_{\nu}<100 \mathrm{eV}$ [2]. Thus a massive $\nu_{r}$ should decay. A very reasonable decay mode to expect would then be:

$$
\nu_{-} \rightarrow \nu_{e, \mu}+\gamma
$$

This mode is the simplest two-body decay that does not violate any known conservation laws (except lepton flavor). No new particles are required and angular momentum sum rules are satisfied.

\section{A Search for Radiative Neutrino Decay}

We are curzently performing a sensitive search for radiative neutrino decay in conjuction with the COMPTEL instrument team. The recently begun search uses type-II supernovae as the source of neutrinos. The high angular resolution and 
sensitivity of the COMPTEL gamma ray telescope [3] provides a unique oportunity to study any supernova gamma ray emissions. By observing SN1987A and extragalactic supernovae, any observed emissions can be analyzed and the relevant neutrino mass and lifetime computed (assuming a radiative decay mode) as described in the following sections. The non-observation of gamma rays from these sources will place significant limits on neutrino mass and lifetime.

\section{Supernova Neutrinos}

The detection of neutrinos from SN1987A [4] confirmed the central theoretical prediction of neutrino production in type-II supernovae: approximately $3 \times 10^{53}$ ergs of binding energy released primarily as neutrinos on a time scale of a few seconds. The neutrinos detected by the IMB and Kamiokande collaborations were very likely $\bar{\nu}_{e}$, based on the relevant neutrino-water cross sections. It is widely believed, however, that since the $\bar{\nu}_{e}$ 's were probably produced by $e^{+} e^{-}$scattering within the proto-neutron star, a roughly equal number of all six neutrino types should have been generated. If the tan neutrino, for example, is composed primarily of a massive component then this "neutrino laboratory", in the form of a type-II supernova, provides a copious number of more than $6 \times 10^{57} \nu_{\tau}$ to study (with an equal number of $\bar{\nu}_{\tau}$ ) [5].

\section{Radiative Neutrino Decay Model}

The computer model developed is a full 3-D simulation including parent neutrino spectra, relativistic kinematics, and angular dependencies. The model allows the neutrinos to stream from the surface of the proto-neutron stai and decay in fight. An expanding spherically-symmetric shell is assumed and relativistic kinematics appropriate to two-body decays in flight are used. Relative time since the $\mathrm{SN}$, resultant gamma ray energies, and angle between the gamma-ray arrival direction and the SN.Earth axis are recorded. In this way, arrival-time distributions can be made on selected gamma-ray energy slices for assumed values of $m_{\nu}$ and $\tau_{\nu}$. In addition, energy spectra can be produced on given time windows along with angular distribution histograms.

\section{Search Method}

Due to the parent neutrino energy spectrum and the assumed finite neutrino lifetime, the energy spectra of the decay gamma rays will evolve over time in a complex (but predictable) manner, examples of which are shown in Figure 1 for two mass values. Because of this spectral evolution, the gamma ray fuence detected - and therefore the mass/lifetime limits obtained - depends on when, in the history of the supernova, an observation occurs. The most sensitive limits can be achieved by observing the source during a period when the gamma ray fluence is 


\section{Radiative Neutrino Decay from Supernovae}
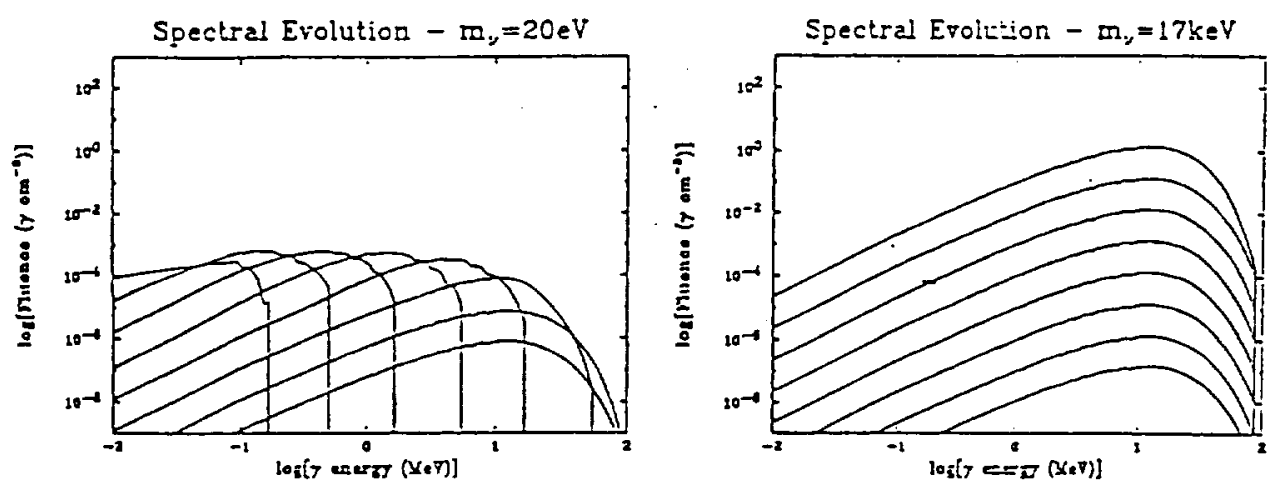

Figure 1: Spectral Evolution $-m_{\nu}=20 \mathrm{eV}$ and $17 \mathrm{keV}, \mathrm{D}=20 \mathrm{Mpc}, \tau_{\nu}=3.5 \times 10^{14}$ $(17 \mathrm{keV}), \tau_{\nu}=5.6 \times 10^{16}(20 \mathrm{eV})$, each curve represents the spectrum for a different time interval. The bottom curve represents the spectrum for arrival times $0-10$ seconds. The second curve for 10-100 seconds, etc.

Table 1: Estimates of Gamma-Ray Flux

\begin{tabular}{||c|c|c|c|c||}
\hline \hline$m_{\nu}$ & $\mathrm{D}$ & $0 \mathrm{~s}$ delay & $10^{5} s$ delay & $10^{7} s$ delay \\
\hline $20 \mathrm{eV}$ & $55 \mathrm{Kpc}$ & 5.6 & 0.0 & 0.0 \\
$17 \mathrm{keV}$ & $55 \mathrm{Kpc}$ & $2.4 \times 10^{4}$ & $2.6 \times 10^{4}$ & $3.5 \times 10^{4}$ \\
$20 \mathrm{eV}$ & $20 \mathrm{Mpc}$ & $1.6 \times 10^{-2}$ & $1.3 \times 10^{-3}$ & 0.0 \\
$17 \mathrm{KeV}$ & $20 \mathrm{Mpc}$ & 0.17 & 0.18 & 0.92 \\
\hline
\end{tabular}

at maximum. The gamma ray arrival times can be distributec over a few seconds or many years, depending on the mass and lifetime of the decaring neutrinos. The arrival time distribution for a sample source is shown in Figure 2.

It is clear that there exists an optimal observational delay wich will provide the most stringent limit. In the current search however, the abilits to choose the optimal delay does not exist. Except for SN1987a observations wijch are an integral part of the Compton observatory's viewing program, we rely on past supernovae - or if we're lucky a new supernova - appearing in the COMPTEL instrument's field of view (approximately $64^{\circ}$ ). Thus, the observational deles since a supernova occurred will dictate the $m_{\nu} / \tau_{\nu}$ parameter space being sampleci in any given observation. Table 1 shows estimates of the gamma ray fluence $\left(\dot{a}\left(\mathrm{~cm}^{-2}\right)\right)$ expected in COMPTEL for different observational delays (assuming an exposure of $5 \times 10^{5} \mathrm{~s}$ ). 


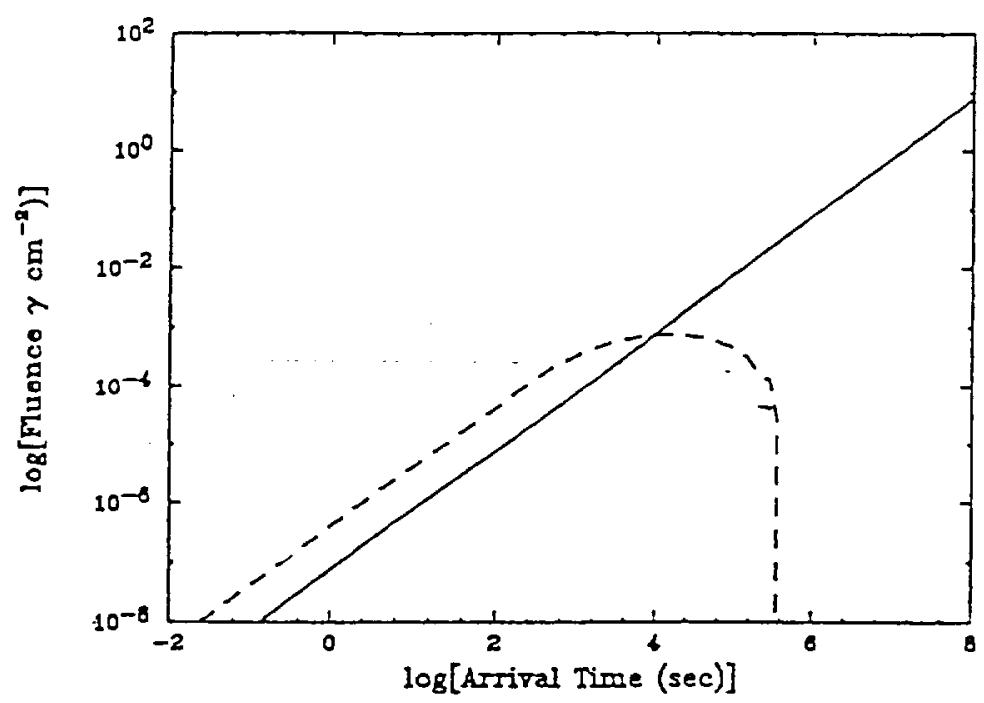

Figure 2: Arrival time distribution of gamma-rays, $D=20 \mathrm{Mpc}, \tau_{\nu}=3.5 \times 10^{14}$ (17keV, solid), $\tau_{\nu}=5.6 \times 10^{16}(20 \mathrm{eV}$,dash $)$

\section{Past Searches}

SN1987A provides a unique opportunity to study neutrino properties. At the time of the supernova there were a number of spacecraft capable of performing gamma ray observations. The most sensitive instrument was the Gamma Ray Spectrometer (GRS) aboard the Solar Maximum Mission (SMM) satellite [6]. No gamma ray pulse was seen within 10 seconds of the IMB neutrino borst time. Thus, limits were set on the neutrino lifetime.

The limits published from the SMM observations are based an a simple 1-D model of radiative neutrino decay using monoenergetic supernova neutrinos. For a light neutrino species the limit from Bludman [7] is $\tau_{\nu}=2.8 \times 10^{15} m_{\nu} \sec$ while for a heavy neutrino the limit is $\tau_{\nu}=6.0 \times 10^{18} \mathrm{~m}_{\nu}^{-1} \mathrm{sec}_{1}$ both assuming a branching ratio to a radiative decay mode of 1 .

Detailed calculations show that COMPTEL, because of improved sensitivity and source exposure, should be able to improve these limits from 1-6 orcers of magnitude for very massive neutrinos, while for light neutrinos the limits achiev. able may be slightly worse (unless a Galactic supernova is detected). Recent anal$y$ sis from the COBE experiment puts very significant limits on the lifetime and branching ratio of radiative neutrino decay [8]. However these results, in addition to other cosmological arguments, require assumptions about the early universe and thus are not a direct test of the stability of massive neutrinos. 
158 Radiative Neutrino Decay from Supernovae

\section{References}

1. H.A.Bethe, Phys. Rev. Lett. 63 (1989) 837; M.Cherry, Nature 347 (1990) 708; J.Bahcall, Neutrino Astrophysics, Cambridge Press, Cambridge (1990);

A.Hime and N.A.Jelly, Oxford preprint (1991); B.Sur, et al., LBL Report (1991); J.J.Simpson, Phys. Rev. Lett. 54 (1985) 1891; J.J.Simpson and A.Hime, Phys. Rev. D39 (1989) 1825; J.J.Simpson and A.Hime, Phys. Rev. D39 (1989) 1837.

2. E.Kolb and M.Turner, The Early Universe, Addison-Wesley, New York (1990) 139.

3. W.Neil Johnson ed., Proceedings of the Gamma Ray Observatory Science Workshop (1989).

4. R.Bionta, et al., Phys. Rev. Lett. 58 (1987) 1494; K.Hirata et al., Phys. Rev. Lett. 58 (1987) 1490.

5. A.Burrows, Univ. of Arizona preprint no. 90-09; D.Kielczewska, Phys. Rev. D41 (1990) 2967.

6. E.Chupp, W.T.Vestrand, and C.Reppin, Phys. Rev. Lett. 62 (1989) 505.

7. S.A.Bludman, to be published Phys. Rev. D (1992).

8. B.B.Nath, Phys. Rev. D 46 (1992) 2341; B.Wang Phys. Rev. D 46 (1992) 2362 . 


\title{
A SEARCH FOR RADIATIVE NEUTRINO DECAY FROM SUPERNOVAE
}

\author{
R.S. Miller and R.C. Svoboda \\ Department of Physics and Astronomy \\ Louisiana State University \\ Baton Rouge, LA 70803-4001
}

Presented by Richard S. Miller

\begin{abstract}
If a massive neutrino species exists then it is possible that it has a radiative decay mode. Cosmological arguments require that a neutrino of mass $>100 \mathrm{eV}$ be unstable. Motivated by these considerations, a 3-D model of radiative neutrino decay has been developed and used to simulate the decay evolution of supernova neutrinos. A sensitive search for the decay gamma rays using extragalactic supernovae and SN1987a as neutrino sources is proceeding using the COMPTEL instrument aboard the Compton Garmma Ray Observatory. The non-observation of decay gamma rays would place more stringent mass/lifetime limits on the neutrino than current limits by about 1-6 orders of magnitude (depending on mass). Such a systematic analysis has never before been attempted In addition, the production of the decay gamma rays over cosmological time-scales is being studied to determine its possible relationship to the diffuse garma ray emission.
\end{abstract}

\section{The Search for Massive Neutrinos}

The search for the existence of a massive neutrino species is an area of intense theoretical and experimental research. Until recently, laboratory studies indicated that the observed properties of the neutrino were consistent with zero rest mass. The notable exceptions have been the experiments studying solar neutrinos and nuclear beta decay.

For over 20 years the study of electron neutrino production in our Sun has led to the so called "Solar Neutrino Problem". So far, all solar neutrino experiments have detected a lower flux than predicted [1]. One compelling explanation for this

111

M. M. Shapiro et al. (eds.). Particle Astrophysics and Cosmology, 111-118.

( 1993 Kluwer Academic Publishers. Prinsed in the Nesherlands. 
is the introduction of neutrino flavor oscillations, which require neutrinos to possess mass [2].

Recently, evidence has been building for the existence of a heavy $17-\mathrm{keV}$ neutrino. This neutrino has been indirectly observed by studying the electron spectra from the beta decay of a number of nuclear isotopes [3]. The deviation in the observed endpoint energies of the beta spectra can be interpreted as the existence of a $17-\mathrm{keV}$ neutrino mixing at the $1 \%$ level with a "normal" massless $\nu_{e}$. Accelerator limits on $\nu_{\mu} \rightarrow \nu_{e}$ oscillations indicate that the massive neutrino is not associated with $\nu_{\mu}[4]$. Since the measurement of the $Z^{\circ}$ width appears to allow only three types of neutrinos with mass less than $45 \mathrm{GeV}$, this massive $17 \cdot \mathrm{keV}$ neutrino must be the $\nu_{r}$. Although the evidence is controversial and awaits unambiguous confirmation the current results make the search for massive neutrinos even more intriguing.

There are also theoretical reasons to believe that neutrinos have mass. Massless neutrinos are not required by the Standard Model of particle physics. In fact it has been necessary to introduce the conserved quantum number of "lepton flavor" to distinguish the three types of neutrinos. This internal degree of freedom might manifest itself in mass differences, as it does for the charged leptons.

Very massive neutrinos ( $>100 \mathrm{eV}$ ) are expected to be unstable. Astrophysical limits based on the requirement that the mass density of the universe not exceed $\Omega=1$ require $\Sigma m_{\nu}<100 \mathrm{eV}$ [5]. Thus a $17-\mathrm{kev} \nu_{\tau}$ should decay. A very reasonable decay mode to expect would then be:

$$
\nu_{\tau} \rightarrow \nu_{e, \mu}+\gamma
$$

This mode is the simplest two-body decay that does not violate any known conservation laws (except lepton flavor). No new particles are required and angtlar momentum sum rules are satisfied.

\section{A Search for Radiative Neutrino Decay}

We are currently performing a sensitive search for radiative neutrino decay in conjuction with the COMPTEL instrument team. The recently begun search uses type-II supernovae as the source of neutrinos. The high angular resolution and sensitivity of the COMIPTEL gamma ray telescope [6] provides a unique oportunity to study any supernova gamma ray emissions. By observing SN1987A and extragalactic supernovae, any observed emissions can be analyzed and the relevant neutrino mass and lifetime computed (assuming a radiative decay mode) as described in the following sections. The non-observation of garmma rays from these sources will place significant limits on neutrino mass and lifetime. 


\section{Supernova Neutrinos}

The detection of neutrinos from SN198TA [i] confirmed the central theoretical prediction of neutrino production in type-II supernovae: approximately $3 \times 10^{53}$ ergs of binding energy released primarily as neutrinos on a time scale of a few seconds. The neutrinos detected by the IMB and Kamiokande collaborations were very likely $\bar{\nu}_{e}$, based on the relevant neutrino-water cross sections. It is widely believed, however, that since the $D_{e}$ 's were probably produced by $e^{+} e^{-}$scattering within the proto-neutron star, a roughly equal number of all six neutrino types should have been generated. If the tau neutrino, for example, is composed primarily of a massive component then this "neutrino laboratory", in the form cf a type-II supernova, provides a copious number of more than $6 \times 10^{57} \nu_{\tau}$ to study (with an equal number of $\nabla_{\mathrm{r}}$ ).

\section{Radiative Neutrino Decay Model}

The computer model developed is a full 3-D simulation including parent neutrino spectra, relativistic kinematics, and angular dependencies. The model allows the neutrinos to stream from the surface of the proto-neutron star and decay in flight. An expanding spherically-symmetric shell is assumed and relativistic linematics appropriate to two-body decays in flight are used. Relative time since the SN, resultant gamma ray energies, and angle between the gamma-ray arrival direction and the SN-Earth axis are recorded. In this way, arrival-time distributions can be made on selected gammarray energy slices for assumed values of $m_{v}$ and $\tau_{\nu}$. In addition, energy spectra can be produced on given time windows along with angular distribution histograms.

The parent neutrino spectrum is a Fermi-Dirac distribution with a temperature of $8 \mathrm{MeV}$ [8]. It is higher than the measured $\nabla_{e}$ temperature ( $4 \mathrm{MeV}$ ) due to the lower opacity of the proto-neutron star to $\nu_{\mu}$ and $\nu_{\text {ras }}$ compared to $\nu_{e}$. The spectrum is normalized such that the total energy in $\nu_{r}$ is equal to the measured neutrino energy released from SN1987a in $\nu_{e}$. This is expected to be true due to equipartition arguments.

\section{Search Method}

Due to the parent neutrino energy spectrum and the assumed finite neutrino lifetime, the energy spectra of the decay garmma rays will evolve over time in a complex (but predictable) manner, examples of which are shown in Figure 1 for two mass values. Because of this spectral evolution, the gamma ray fluence detected - and therefore the mass/lifetime limits obtained - depends on when. in the history of the supernova an observation occurs. The most sensitive limits can be 

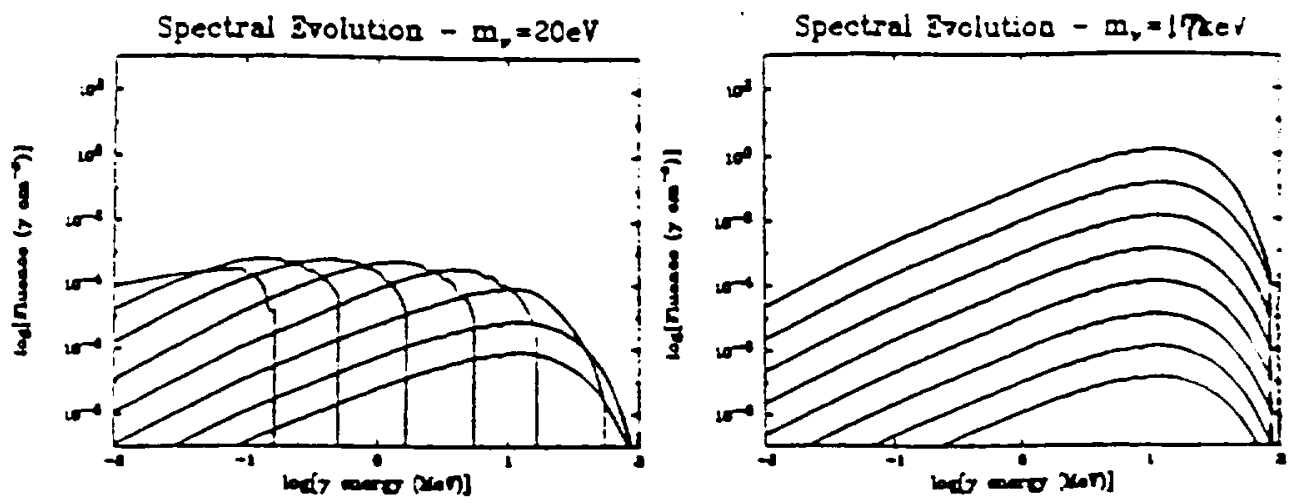

Figure 1: Spectral Evolution - $m_{\nu}=20 \mathrm{eV}$ and $17 \mathrm{keV}, \mathrm{D}=20 \mathrm{Mpc}, \tau_{\nu}=3.5 \times 10^{14}$ $(17 \mathrm{keV}), \tau_{\nu}=5.6 \times 10^{16}(20 \mathrm{eV})$, each curve represents the spectrum for a different time interval. The bottom curve represents the spectrum for arrival times 0-10 seconds. The second curve for $10-100$ seconds, etc.

achieved by observing the source during a period when the gamma ray fluence is at maximum. The gamma ray arrival times can be distributed over a few seconds or many years, depending on the mass and lifetime of the decaying neutrinos. The arrival time distribution for a sample source is shown in Figure 2.

It is clear that there exists an optimal observational delay which will provide the most stringent limit. In the current search however, the ability to choose the optimal delay does not exist. Except for SN1987a observations which are an integral part of the Compton observatory's viewing program, we rely on past supernovae - or if we're luchy a new supernova - appearing in the COMPTEL instrument's field of view (approximately $64^{\circ}$ ). Thus, the observational delay since a supernova occurred will dictate the $m_{\nu} / \tau_{\nu}$ parameter space being sampled in any given observation. Table 1 shows estimates of the gamma ray fluence $\left(\phi_{\gamma}\left(\mathrm{cm}^{-2}\right)\right)$ expected in COMPTEL for different observational delays (assuming an exposure of $5 \times 10^{5} \mathrm{~s}$ ).

\section{Past Searches}

No systematic search of this type has been attempted before. However, SN1987A provided a unique opportunity to study neutrino properties. At the time of the supernova there were a number of spacecraft capable of performing gamma ray observations. The most sensitive instrument was the Gamma Ray Spectrometer (GRS) aboard the Solar Maximum Mission (SMM) satellite [9]. No gamma ray pulse was seen within 10 seconds of the IMB neutrino burst time. Thus, limits 


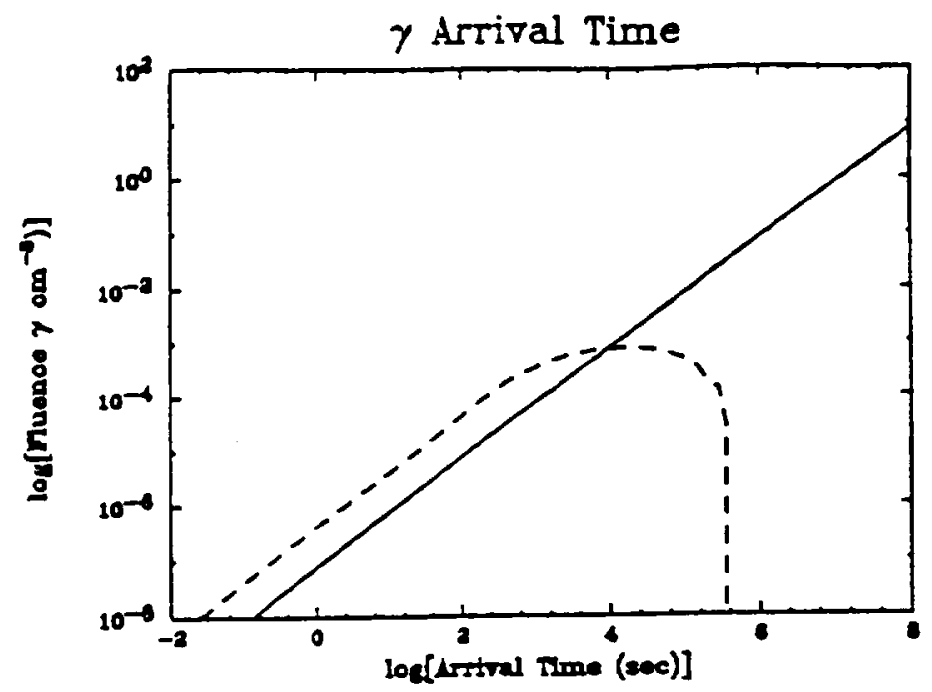

Figure 2: Arrival time distribution of gamma-rays, $\mathrm{D}=20 \mathrm{Mpc}, \tau_{\nu}=3.5 \times 10^{14}$ (17keV,solid), $\tau_{\nu}=5.6 \times 10^{16}(20 \mathrm{eV}$,dash)

Table 1: Estimates of Gamma-Ray Flux

\begin{tabular}{||c|c|c|c|c||}
\hline \hline$m_{v}$ & $\mathrm{D}$ & 0 s delay & $10^{5}$ s delay & $10^{7}$ s delay \\
\hline $20 \mathrm{eV}$ & $55 \mathrm{Kpc}$ & 5.6 & 0.0 & 0.0 \\
$17 \mathrm{keV}$ & $55 \mathrm{Kpc}$ & $2.4 \times 10^{4}$ & $2.6 \times 10^{4}$ & $3.5 \times 10^{4}$ \\
$20 \mathrm{eV}$ & $20 \mathrm{Mpc}$ & $1.6 \times 10^{-2}$ & $1.3 \times 10^{-3}$ & 0.0 \\
$17 \mathrm{KeV}$ & $20 \mathrm{Mpc}$ & 0.17 & 0.18 & 0.92 \\
\hline
\end{tabular}


were set on the neutrino lifetime.

The limits published from the SMM observations are based on a simple 1-D model of radiative neutrino decay using monoenergetic supernova neutrinos. For a light neutrino species the limit from Bludman [10] is given by:

$$
\tau_{\nu}=2.8 \times 10^{15} \mathrm{~m}_{\nu} \mathrm{sec}
$$

while for a heavy neutrino the limit is:

$$
\tau_{\nu}=6.0 \times 10^{18} \mathrm{~m}_{\nu}^{-1} \mathrm{sec}
$$

both assuming a branching ratio to a radiative decay mode of 1 .

As suggested above, the parameter space sampled in such a search is very sensitive to the observational delay and source exposure. Since the supernova occurred. Since the observation window of SN1987a by SMM was short (only 10 seconds due to calibration runs, the SAA, and background considerations), and the observational delay was zero, the sensitivity for observing gamma rays for very massive neutrinos is reduced.

\section{Neutrino Decay and the Diffuse Gamma Ray Emission}

Evidence for a diffuse garmms ray flux comes primarily from SAS 2 data [11]. There appear to be multiple components to the measured flux: a galactic and isotropic components. The galactic component is thought to originate from cosmic ray electron brehmstrahlung or Compton radiation, while the origin of the isotropic component is still a significant open question. Many ideas for the production of this isotropic component have been put forward, although none are completely satisfactory [12]. We are currently investigating the role radiative neutrino decay would play in the production of the isotropic diffuse emission.

Assuming an isotropic and homogeneous universe, the energy spectrum of gamma ray emission (due to raciative neutrino decay) from all type-ll supernovae since the onset of galaries can be computed. The shape of the spectrum is dependent on $m_{\nu}$ and $\tau_{\nu}$, as well as the estimated supernova rate per galaxy type, and the density of galaxies in the Universe. By comparing spectral shapes limits can be placed on $m_{\nu}$ and $\tau_{\nu}$, while absolute normalization of the spectrum will place limits on the radiative decay branching ratio. The results of this investigation are forthcoming. 


\section{Summary}

This search and analysis is extremely timely due to the recent developments in particle and astrophysics. No systematic study of this type has been attempted before; a non-observation of the gamma ray products from neutrino decay would place significant limits on the mass and lifetime of neutrinos while observation of this process would be a scientific triumph with profound iroplications for the Standard Model of particle physics, many astrophysical processes, and cosmology.

The time difference between a supernova and the beginning of its observation by COMPTEL will determine the mass/lifetime parameter space sampled. In addition, the source exposure, energy spectra of detected gamma rays, and arrival times will permit analysis of COMPTEL data to look for neutrino decay properilies with a sensitivity unobtainable in terrestrial laboratories.

\section{References}

1. H.A.Bethe, Phys. Rev. Lett. 63 (1989) 837; M.Cherry, Nature 347 (1990) 708.

2. J.Bahcall, Neutrino Astrophysics, Cambridge Press, Cambridge (1990).

3. A.Hime and N.A.Jelly, Oxford preprint (1991); B.Sur, et al., LBL Report (1991); J.J.Simpson, Phys. Rev. Lett. 54 (1985) 1891; J.J.Simpson and A.Hime, Phys. Rev. D39 (1989) 1825; J.J.Simpson and A.Hime, Phys. Rev. D39 (1989) 1837.

4. L.A.Ahrens, et al., Phys. Rev. D31 (1985) 2732; M.J.Dugan, A.V.Monohar, and A.Nelson, Phys. Rev. Lett. 53 (1985) 170.

5. E.Kolb and M.Tumer, The Eariy Universe, Addison-Wesley, New York, (1990) 139.

6. W.Neil Johnson ed., Proceedings of the Gamma Ray Observatory Science Workshop (1989).

7. R.Bionta, et al., Phys. Rev. Lett. 58 (1987) 1494; K.Hirata et al., Phys. Rev. Lett. 58 (1987) 1490. 
8. A.Burrows, Univ. of Arizona preprint no. 90-09; D.Kielczewska, Phys. Rev. D41 (1990) 2967.

9. E.Chupp, W.T.Vestrand, and C.Reppin, Phys. Rev. Lett. 62 (1989) 505.

10. S.A.Bludman, to be published Phys. Rev. D (1992).

11. C.E.Fichtel, G.A.Simpson,D.J.Thompson, Ap.J. 222 (1979) 833.

12. P.V.Ramana Murthy and A.W.Wolfendale, Gamma-ray Astronomy, Cambride Press, Cambridge (1986). 